\title{
Some Aspects of the BEM Research in China
}

\author{
Zhenhan Yao and Qinghua Du \\ Department of Engineering Mechanics, Tsinghua University, Beijing, China
}

\begin{abstract}
In this paper, a short historical review of the BEM research in China is given first. The investigation on the BEM in China was started in 1978 at the Tsinghua University, based on the pioneering work published by Frank Rizzo [F. J. Rizzo, An integral equation approach to boundary value problems of classical elastostatics, Quart. Appl. Math., 25, 83-95 (1967)] and others. During the last 25 years, we have organized six national conferences on BEM, two international conferences and eight China-Japan/Japan-China symposia on BEM. The BEM investigations in the authors' group at Tsinghua are briefly introduced next. Some representative investigations by other groups in Mainland China are also introduced. The related papers and other publications are listed in the References.
\end{abstract}

\section{Historical Review}

In China, we started the investigation on boundary element methods in 1978 at Tsinghua University. At first, the second author, Du, introduced the related papers published by Frank Rizzo, T. A. Cruse and other BEM pioneers to the researchers in China, and suggested to them to pay attentions on the development of the boundary integral equation (BIE) method. In the authors' group, we started with the elastic stress concentration problems using boundary integral equation - boundary element method, including axi-symmetric body with symmetric and asymmetric loading, and also three-dimensional elasticity problems. Another topic is the plate bending problems, which related to higher order differential operator. At that time, the paper of Frank Rizzo published in 1967 was the first literature on BEM available to us. In the first several years, we learnt that the well-known kernel functions can be generalized by superposition, integration and differentiation to obtain some useful new kernel functions for different problems, such as the torsion of circular shaft with variable diameters, axi-symmetric problems, plate bending problems and crack problems. In 1982, we published our work at Acta Mechanica Solida Sinica in Chinese ${ }^{[1]}$, and gave a workshop to more than 100 audiences organized by the editorial board of that journal. In 1982, Du attended the Fourth International Conference on Boundary Element Methods in Southampton ${ }^{[2]}$, and in 1984 we published a paper on plate bending problem in the international journal Applied Mathematical Modelling ${ }^{[3]}$. At the same time some Chinese scholars started BEM research abroad and returned to China. More and more people started to join the research on boundary element methods.

In 1985, Du organized the First National Conference on BEM in Chongqing together with Jialin Zhu. This series of conferences took place every three years: 1988, organized by Du and Rong Qin in Nanning; 1991, Du and Weifang Zhong in Wuhan; 1994, Du and Hongdao Jiang in Nanjing; 1997, the first author, Yao, organized in Beijing; 2000, Yao and Jialin Zhu organized in Chongqing again. G. Maier from Italy also attended the 2000 conference and gave a lecture on Galerkin BEM. In 1980s the number of participants of 
these conferences was more than 100. The maximum number, in 1988, exceeded 150. In recent years, the number of participants of such conferences was about 40. However, we have decided to continue such conference series, because the investigation on BEM is being developed to deal with more difficult problems, and some new developments show good prospers of the BEM, such as the fast multipole BEM, and other efficient methods. The next conference in 2003, will be organized by Yao and Guangxian Shen in Qinhuangdao, and combined with the Global Chinese Workshop on Boundary Element and Meshless Methods.

On the other hand, we initiated and promoted many international exchanges for the BEM research. In 1986, Du organized an International Conference on Boundary Element Methods in Beijing ${ }^{[4]}$. More than 20 participants from abroad attended that conference, including S. Mukherjee, G. C. Hsiao from United States, S. Kobayashi, M. Tanaka from Japan. In 1987, Du cooperated with Masataka Tanaka started the China-Japan/Japan-China Symposium on Boundary Element Methods, in Karuizawa, $\operatorname{Japan}^{[5]}$, and then, 1988 in Beijing China ${ }^{[6]}, 1990$ in Hachiohji Japan ${ }^{[7]}, 1991$ in Beijing China $^{[8]}, 1993$ in Sapporo Japan ${ }^{[9]}$, and 1994 in Shanghai China ${ }^{[0]}$. In 1996, Masataka Tanaka and Yao organized the seventh symposium in Fukuoka Japan ${ }^{[11]}$, and then 1997 in Beijing China ${ }^{[12]}$. Recently, Yao and M. H. Aliabadi organized the Third International Conference on Boundary Element Techniques ${ }^{[13]}$, more than 50 participants attended that conference, including 30 from abroad.

\section{BEM Investigations by the Authors' Group}

As mentioned above, we started the BEM investigation on elastic stress concentration and plate bending problems. Based on the Rizzo's direct boundary integral equation boundary element method formulation, we generalized the kernel function in BIE for the problems with symmetry by superposition, for the problems of axisymmetric body under torsion, axisymmetric loading, or pure bending by integration, and for a highly accurate scheme of plate bending problems by differentiation.

Because of the advantages of BEM and FEM, some schemes combined BE and FE had been suggested in our group, by Zhangzhi Cen and $\mathrm{Du}^{[14]}$. BE - FE coupling approaches investigated are not only for the elastic problems, but also for elasto-plastic problems. We also combined BEM and modal synthesis method for the modal analysis of $2 \mathrm{D}$ elastic solids ${ }^{[15]}$.

Based on the BEM investigation on elasticity problems, we also investigated some inverse problems, including shape optimization ${ }^{[16]}$, and defect identification ${ }^{[17]}$. Some numerical examples of practical engineering problems on shape optimization shown some distinctive advantages of the boundary element method.

In our group, Zhangzhi Cen had cooperated with G. Maier, and worked on the symmetric Galerkin $\mathrm{BEM}^{[18]}$, structural limit analysis ${ }^{[19]}$ and shake-down analysis ${ }^{[20]}$. He also did work on the stress intensity factor computation by $\mathrm{BEM}^{[21]}$.

For the integration of kernel and shape function product on an element, we applied the Gaussian quadrature with equiaccuracy approach: the number of Gauss integration points 
is determined by the prescribed accuracy of integration. For the treatment of singular or nearly singular integrals, a scheme using several known specific solutions was suggested $^{[22]}$.

For the error estimations, a direct error estimator was proposed ${ }^{[23]}$, which is based on the differences of boundary conditions, namely the difference of the given values for the boundary conditions and the limit of corresponding continuous domain variables as the point approaches boundary. For the displacement given boundary, such error estimator is quite simple.

For contact problems, element to element comforming contact scheme has been adopted. And for 2D and 3D moving contact problems, schemes of comforming boundary elements were suggested ${ }^{[24,25]}$, in which the boundary nodes are divided into fixed nodes and movable nodes, and the contact boundary conditions can still be satisfied exactly in the discretization sense. In this way, the advantage of high accuracy in BEM can be preserved.

As composite materials have been applied widely in engineering practice, we paid our attention to the simulation of composite materials with BEM. A scheme of repeated similar sub-region approach was developed to deal with the simulation of 2D solids with randomly distributed inclusions ${ }^{[26]}$. We also used the repeated similar sub-region approach to simulation the behavior of heterogeneous materials ${ }^{[27]}$.

For the application of BEM to practical engineering problems, we also investigated parallel algorithm of BEM on PC cluster ${ }^{[28]}$. In 2000 we computed on a cluster consisted of 8 PCs, some engineering stress analysis problems, with the maximum size of the problem being $45,000 \mathrm{DOF}$.

In recent years, the fast multipole BEM show prosperous future on dealing with practical engineering and scientific problems. We started with the simulation of 2D solids with a large number of inclusions, in the preliminary stage; we already simulated the problem with 1,600 inclusions, and with $544,000 \mathrm{DOF}^{[29]}$.

Based on the boundary integral equation, we also presented a boundary type meshless method, called hybrid boundary node method ${ }^{[30,31]}$. The schemes for $2 \mathrm{D}$ and $3 \mathrm{D}$ potential and elasticity problems have been presented.

In the near future, we will pay much attention on the large scale engineering practice problems using fast multipole BEM and boundary type meshless methods, including local nonlinear problems combined with DRBEM, thin structure analysis, multi-physics field problems and multi-scale problems in MEMS and NEMS.

\section{Some Representative BEM Investigations by Other Groups in China}

At Tsinghua University, besides our group in the Department of Engineering Mechanics, there is another group in the Department of Hydraulic and Hydropower Engineering. Chuhan Zhang, et al, investigated the dynamic interaction of dam and foundation ${ }^{[32]}$, presented some efficient schemes, such as infinite boundary element approach ${ }^{[33]}$. They applied the boundary element, infinite boundary element and finite element coupled to 
deal with practical engineering problems in the design of dams and achieved important progress.

Haichang Hu at Peking University, who is well-known for the Hu-Washizu variational principle, also carried out in-depth study on the BIE and BEM. He found that the conventional boundary integral equations for $2 \mathrm{D}$ potential and elasticity problems are not equivalent to the boundary value problem of corresponding partial differential equation. $\mathrm{He}$ and his co-authors at Peking University and Zhejiang University, presented the sufficient and necessary boundary integral equation equivalent to the partial differential equation for the boundary value problems ${ }^{[34,35]}$.

On the mathematical aspects, Dehao $\mathrm{Yu}$ and Kang Feng, from the Institute of Computational Mathematics, Chinese Academy of Sciences, presented the mathematical theory of natural boundary element method ${ }^{[36,37]}$, which has some attractive mathematical features.

Jialin Zhu from Chongqing University and Houde Han from Tsinghua University, in the department of mathematics, also carried out the investigations on mathematical aspects of $\mathrm{BEM}^{[38,39]}$.

Xing Ji and his group, originally at Xi'an Jiaotong University, afterwards moved to Tongji University, carried out systematic investigations on boundary element methods, especially on elasto-dynamics problems by Laplace transformation or Fourier eigen-transformation, 2D and axisymmetric elasto-plastic finite deformation problem, rigid body - fluid coupling problems, and so on ${ }^{[40-42]}$.

In Huazhong University of Sciences and Technology, Yuying Huang and his group carried out systematic investigations on fluid - structure interaction problems by boundary element method ${ }^{[43]}$; Weifang Zhong and his group, on wave propagation problems $^{[44]}$.

Zhenxing Feng from Wuhan University investigated moving boundary problems by boundary element methods, and some numerical algorithms in $\mathrm{BEM}^{[45]}$. He found that BEM has some advantages for such problems.

Guangxian Shen and his group in Yanshan University investigated contact problem in rolling process thoroughly, from elastic to elasto-plastic and finite deformation, and achieved good results for the practical engineering problems ${ }^{[46,47]}$.

Maokuang Huang and his former students, from University of Sciences and Technology of China carried out investigations on plate bending problems and elasticity problems by different boundary element methods, and presented some efficient schemes ${ }^{[48,49]}$.

Youcheng Wang and his former students investigated plate bending problems by boundary element method and presented some efficient schemes ${ }^{[50]}$.

Tianqi Ye and his group at Northwestern Polytechnical University investigated the BEM for nonlinear plate bending problems, numerical techniques in BEM, adaptive BEM, parallel algorithms, and applied them to some practical engineering problems ${ }^{[11,52]}$.

Hongdao Jiang and his group also carried out the investigations on the numerical aspects and engineering applications of $\mathrm{BEM}^{[53]}$. Rong Qin presented the Spline boundary 
element method, and applied to different engineering problems ${ }^{[54]}$.

The above mentioned work are only a partial list of investigations on BEM in Mainland China. The work done by Chinese authors, who worked or studied outside of Mainland China, are not included.

Most of the authors, who started BEM investigations around 1980, are already older than 60 years. Now more younger scholars in China are actively involved in the BEM research. We are determined to continue the research on BEM and the exchanges in the BEM community, and strengthen the international exchanges in the BEM research.

\section{References}

[1] $\mathrm{Du} \mathrm{QH}$, Yao ZH, Some basic problems and engineering applications of BIE-BEM in elasticity. Acta Mechanica Solida Sinica, 3: 1-12, 1982 (In Chinese).

[2] $\mathrm{Du} \mathrm{QH}$, Yao ZH, Application of BEM to 2-D and 3-D stress analysis and plate bending problem in elasticity. Proc. of BEM IV, Southampton, Springer-Verlag, 1982.

[3] $\mathrm{Du} \mathrm{QH}$, Yao $\mathrm{ZH}$, Song GS, Solution of some plate bending problems using the boundary element method. Applied Mathematical Modelling, 8: 15-22, 1984.

[4] Du QH ed., Boundary Elements. Pergamon Press, 1986.

[5] Tanaka M, Du QH eds., Theory and Applications of Boundary Element Methods. Pergamon Press, 1987.

[6] Du QH, Tanaka M eds., Theory and Applications of Boundary Element Methods. Tsinghua University Press, 1988.

[7] Tanaka M, Du QH eds., Theory and Applications of Boundary Element Methods. Pergamon Press, 1990.

[8] Du QH, Tanaka M eds., Theory and Applications of Boundary Element Methods. International Academic Publishers, 1991.

[9] Tanaka M, Du QH, Honma T eds., Theory and Applications of Boundary Element Methods. Elsevier, 1993.

[10] Du QH, Tanaka M, Ji X eds., Theory and Applications of Boundary Element Methods. International Academic Publishers, 1994.

[11] Tanaka M, Yao ZH eds., Theory and Applications of Boundary Element Methods. Elsevier, 1996.

[12] Yao ZH, Tanaka M eds., Theory and Applications of Boundary Element Methods. International Academic Publishers, 1997.

[13] Yao ZH, Aliabadi MH eds., Boundary Element Techniques. Tsinghua University Press, Springer-Verlag, 2002.

[14] Cen ZZ, Wang XC, Du QH, Elastoplastic analysis by the coupling of boundary element method to finite element method. Journal of Tsinghua University, 28: 34-43, 1988. (In Chinese)

[15] Du QH, Yao ZH, Cen ZZ, Eigenproblems by boundary element method using component mode synthesis. In [8].

[16] Song GS, Du QH, Optimization for some elastic structures by boundary element method. Proc. of BETech 10, Comp. Mech. Pub. 243-257, 1990.

[17] Yao ZH, Gong B, Defect identification using boundary element methods of 
elastostatics. In Bui HD et al eds., Inverse Problems in Engineering Mechanics, Balkema, 387-392, 1994.

[18] Cen ZZ, Maier G, Novati G, Stability theory of cohesive crack model - discussion. Journal of Engineering Mechanics - ASCE, 120: 1168-1170, 1994.

[19] Liu YH, Cen ZZ, Xu BY, A numerical method for plastic limit analysis of 3-D structures, Int. J. Solids Structures, 32: 1645-1658, 1995.

[20] Zhang XF, Liu YH, Cen ZZ, A solution procedure for lower bound limit and shakedown analysis by SGBEM, Acta Mechanics Solida Sinica, 14: 118-129, 2001.

[21] Chen TZ, Wang B, Cen ZZ, A symmetric Galerkin multi-zone boundary element method for cohesive crack growth. Engineering Fracture Mechanics, 63: 591-609, 1999.

[22] Huo TR, Yao ZH, Evaluation of mechanical variables at boundary and near boundary points of elastic structures by BEM, Numerical Computation and Computer Application, 14(1), 1993 (In Chinese).

[23] Yao ZH, Dong CY, A direct error estimator and adaptive scheme of boundary element method. In [8].

[24] Yao ZH, Pu JP, Moving and rolling contact of 2D elastic bodies with defects using boundary element method. In Dominquez J and Brebbia CA eds: Computational Methods in Contact Mechanics V, WIT Press, 283-292, 2001.

[25] Liu YJ, Yao ZH, Boundary element method for moving contact of 3D elastic bodies. In [13].

[26] Yao ZH, Wang PB, Kong FZ, Simulation of 2D elastic solids with randomly distributed inclusions. In [13].

[27] Lin CY, Yao ZH, Simulation of elastic heterogeneous material by similar subdomain approach of BEM. In [13].

[28] Yin X, Cheng JG, Yao ZH, Solving large-scale BEM problems in a cluster environment. Proc. Of 4th Int. Conf. on High Performance Computing in Asia-Pacific Region, Beijing, China, 2000.

[29] Wang HT, Yao ZH, Application of fast multipole BEM for 2D elastic body with circular inclusions. In [13].

[30] Zhang JM, Yao ZH, A hybrid boundary node method. Int. J. Num. Meth. Engrg, 53: 751-763, 2002.

[31] Zhang JM, Yao ZH, A meshless regular hybrid boundary node method. Computer Modeling in Engineering and Sciences, 2: 307-318, 2001.

[32] Zhang CH, Chen XF, Wang GL, A coupling model of FE-BE-IE-IBE for non-linear layered soil-structure interactions. Earthquake Eng. Struc. 28: 421-441, 1999.

[33] Zhang CH, Song CM, Pekau, Infinite boundary elements for dynamic problems of 3-D half space. Int. J. Numer. Meth. Engrg, 31: 447-462, 1991.

[34] Hu HC. A new type of boundary integral equation in elasticity. Scientia Sinica, 30(4), 1987.

[35] He WJ, Ding HJ, Hu HC, A necessary and sufficient boundary integral formulation for plane elasticity. Commun. Numer. Meth. Engrg, 12: 413-424, 1996.

[36] Feng K, Yu DH, Canonical integral equations of elliptic boundary value problems and their numerical solutions. Proc. China-France Symp. On the Finite Element Method, 1982, Beijing, Science Press, 1983.

[37] Yu DH, The Mathematical Theory of Natural Boundary Element Method. Science 
Press, 1993 (In Chinese).

[38] Zhu JL, Domain decomposition method with boundary elements. In [9].

[39] Han HD, A boundary element approximation of a Signorini problem with friction obeying Coulomb law. J. Comput. Math., 12: 147-162, 1994.

[40] Cheng YM, Ji X, An investigation on a new integral transform boundary element method for elastodynamics. Acta Mechanica Solida Sinica, 10: 246-254, 1997.

[41] Chen ZQ, Ji X, Boundary element analysis of finite deformation problems of elastoplasticity. In [5].

[42] Zang YL, Ji X, A boundary element method for small-amplitude viscous fluid sloshing in couple with structural vibration. Acta Mechanica Solida Sinica, 12: 9-21, 1999.

[43] Huang YY, Yue DY, Qian Q, A time domain boundary element method for water-solid impact analysis. Acta Mechanica Solida Sinica, 8: 337-348, 1995.

[44] Liu XG, Zhong WF, Chen BH, A boundary element method for calculating elastic waves scattered by axisymmetric inclusion. Acta Mechanica Solida Sinica, 11: 359-364, 1998.

[45] Feng ZX, Tang SW, Li ZX, A new time marching BE scheme for transient heat transfer with a thermal moving boundary. Eng. Anal. Bound. Elem., 22: 71-75, 1998.

[46] Shen GX, Shu XD, Li M, The 3-D boundary element method of roller bearing by plate element analogue. Acta Mechanica Solida Sinica, 14: 268-274, 2001.

[47] Liu DY, Shen GX, Three-dimensional elastic multipole BEM and computation of roller deformation field. In [13].

[48] Huang MK, Lei XY, On the problem of elastic torsion of variable diameter circular shafts by the boundary integral equation method, Computer and Structures, 19(3), 1984.

[49] Chen HB, Lu P, Huang MG, Williams FW, An effective method for finding values on and near boundaries in the elastic BEM. Computers and Structures, 69: 421-431, 1998.

[50] Wang YC, Liu Z, Wu Y, All particular solution method in boundary element techniques. In [10].

[51] Ye TQ, Liu YJ, Finite deflection analysis of elastic plates by boundary element method. Applied Mathematical Modelling, 9: 183-188, 1985.

[52] Lu S, Ye TQ, Direct evaluation of the singular integrals in elastoplastic analysis by BEM. Int. J. Numer. Meth. Engrg, 32: 295-311, 1991.

[53] Liu HB, Jiang HD, Statistical analysis for response of probabilistic structures. In [10].

[54] Qin R, Fundamentals and application of spline BEM. Proc. of BEM V, Springer-Verlag, 1983. 